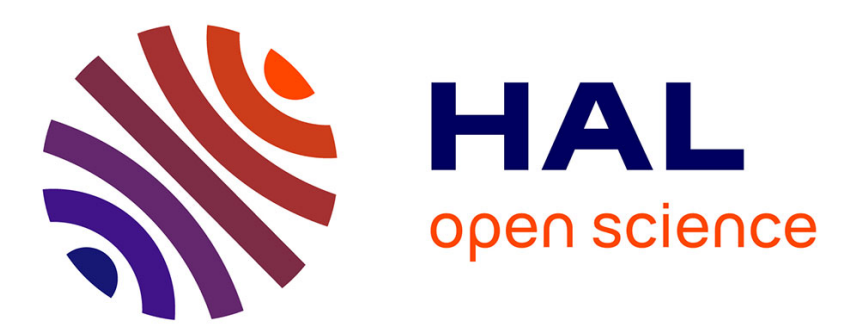

\title{
Particle Filter-Based Model for Online Estimation of Demand Multipliers in Water Distribution Systems under Uncertainty
}

Nhu Cuong Do, Angus Simpson, Jochen Deuerlein, Olivier Piller

\section{- To cite this version:}

Nhu Cuong Do, Angus Simpson, Jochen Deuerlein, Olivier Piller. Particle Filter-Based Model for Online Estimation of Demand Multipliers in Water Distribution Systems under Uncertainty. Journal of Water Resources Planning and Management, 2017, 143 (11), 04017065, 15 p. 10.1061/(ASCE)WR.1943-5452.0000841 . hal-01807802

\section{HAL Id: hal-01807802 https://hal.science/hal-01807802}

Submitted on 5 Jun 2018

HAL is a multi-disciplinary open access archive for the deposit and dissemination of scientific research documents, whether they are published or not. The documents may come from teaching and research institutions in France or abroad, or from public or private research centers.
L'archive ouverte pluridisciplinaire HAL, est destinée au dépôt et à la diffusion de documents scientifiques de niveau recherche, publiés ou non, émanant des établissements d'enseignement et de recherche français ou étrangers, des laboratoires publics ou privés. 

distribution systems under uncertainty

$4{ }^{1}$ - School of Civil, Environmental and Mining Engineering, University of Adelaide, Adelaide SA 5005,

$5 \quad$ Australia.Email:nhu.do@adelaide.edu.au. *-Corresponding author

62 - Professor, School of Civil, Environmental and Mining Engineering, University of Adelaide, Adelaide SA

7 5005, Australia.Email: angus.simpson@adelaide.edu.au

83 - Senior Researcher, 3S Consult GmbH, Karlsruhe, Germany.Email: deuerlein@,3sconsult.de

$9{ }^{4}$ - Senior Research Scientist, Irstea, UR ETBX, Department of Waters, France, Bordeaux. Email: 10 olivier.piller@irstea.fr

\section{Abstract}

12 Accurate modeling of water distribution systems is fundamental for the planning and operating 13 decisions in any water network. One important component that directly affects model accuracy is

14 the knowledge of nodal demands. Conventional models simulate flows and pressures of a water 15 distribution network either assuming constant demands at nodes or using a short-term sample of 16 demand data. Due to the stochastic behavior of the water demands, this assumption usually leads 17 to an inadequate understanding of the full range of operational states in the water system. 18 Installation of sensor devices in a network can provide information about some components in the 19 system. However, the requirement for a reliable water distribution model that can assist with 20 understanding of real-time events in the entire water distribution system is still an objective for 21 hydraulic engineers.

22 This paper proposes a methodology for the estimation of online (near real-time) demand 23 multipliers. A predictor-corrector approach is developed which predicts the hydraulic behaviors of 24 the water network based on a nonlinear demand prediction model, and corrects the prediction by 
25 integrating online observation data. The standard particle filter and an improved particle filter

26 method, which incorporates the evolutionary scheme from genetic algorithms into the resampling

27 process to prevent particle degeneracy, impoverishment and convergence problems, are

28 investigated to implement the predictor-corrector approach. Uncertainties of model outputs are

29 also quantified and evaluated in terms of confidence intervals. Two case studies are presented to

30 demonstrate the effectiveness of the proposed particle filter model. Results show that the model

31 can provide a reliable estimate of demand multipliers in near real-time contexts.

33 Keywords: Particle filters, sequential Monte Carlo method, real-time demand estimation, water

34 distribution systems, uncertainty.

\section{Introduction}

36 Water distribution systems (WDS) are constructed to supply water for domestic, industrial and

37 commercial consumers. The design, operation and management of these distribution systems is

38 usually supported by the application of hydraulic models, which are built to replicate the behavior

39 of real systems. These conventional models simulate flows and pressures of a WDS either under

40 steady state conditions (constant demands and operational conditions) or under a short term

41 extended period simulation (time-varying demands and operational conditions), for example a day

42 or a week (USEPA 2005). The outputs from hydraulic models, therefore, usually represent the

43 distribution system behavior during the sampling period (Preis et al. 2009). This leads to an

44 inadequate understanding of the full range of operational states in the water system.

45 The installation of sensor devices as well as the Supervisory Control and Data Acquisition

46 (SCADA) systems within the WDS can provide information on the status of some components in

47 the system. However, the use of this additional data is currently limited to computing gross 
differences between the model outputs and reality (Kang \& Lansey 2009). Modification of the hydraulic models to maintain the consistency between observed data and simulated data is still a challenge that needs to be dealt with. Estimation of the model states/parameters, hence, is required

51 so that the model is able to represent the real system.

52 Estimation is the process of fitting the outputs from the computer model, usually the pressures and

53 flow rates at particular locations in the water network, with the field measurements, in order to 54 calculate unknown variables of interest. Initial estimation studies in WDSs were pioneered by 55 Rahal et al. (1980), Walski (1983) and Bhave (1988) with the proposal of the ad hoc (trial-anderror) calibration schemes, in which an iterative process to update unknown model parameters was

57 implemented. Due to the slow convergence rate, this method is only applicable to small water 58 networks. Later, explicit calibration methods were introduced (Ormsbee \& Wood 1986; Boulos \& Wood 1990; Boulos \& Ormsbee 1991). These methods solved an even-determined set of water network equations where the number of unknown parameters is grouped to be equal to the number

61 of measurements. As the measurement errors were also neglected, these methods usually did not 62 represent real-world practical outputs. Therefore, explicit calibration models were often used to 63 analyse historic events in water systems (Savic et al. 2009). Subsequently, implicit methods were 64 developed using either mathematical techniques or evolutionary optimization techniques, for 65 example: Complex Method (Ormsbee 1989), Weighted Least Squares approaches (Lansey \& 66 Basnet 1991; Datta \& Sridharan 1994), Singular value decomposition (SVD) method (Sanz \& 67 Pérez 2015) or Genetic Algorithms (GA) (Preis et al. 2009; Abe \& Peter 2010; Do et al. 2016). 68 These methods have drawn a high degree of attention from researchers. However, these models 69 are mostly impractical due to either a requirement for a large quantity of 'good' observation data 70 (Savic et al. 2009) or ignoring model uncertainties. Furthermore, few approaches have attempted 
71 to estimate model parameters and model states in conjunction with model uncertainties. Bargiela

72 and Hainsworth (1989) found that a good approximation of pressure uncertainty bounds can be

73 obtained by a linearization of the mathematical network model. Piller (1995) and Bush and Uber

74 (1998) used a sampling design method to estimate the model parameters and approximate the uncertainties. Lansey et al. (2001) applied a first-order approximation method to identify pipe roughness uncertainty. Nagar and Powell (2002) applied a linear fractional transformation and semi-definite programming method to estimate the pressure heads and their confidence bounds. In addition, some probabilistic methods (Xu \& Goulter 1998; Kapelan et al. 2007; Hutton et al. 2013) have also been investigated for the estimation of model parameters. Due to the complexity of the uncertainties, estimation methods associated with uncertainty quantification are still a continuing

81 research area, especially for real-time estimation purposes.

82 The complexity of uncertainties in WDS modeling has been addressed in Hutton et al. (2012b), in

83 which the uncertainty is divided into three categories: (1) structural uncertainty, (2) parameter

84 uncertainty and (3) measurement/data uncertainty. Structural uncertainty derives from the 85 mathematical representation of the real system, such as network skeletonization and model 86 aggregation. Skeletonized and/or aggregated models are predominantly used instead of all-pipes 87 models to reduce the complexity of the network being analysed as well as to increase 88 computational speed. It has been shown that skeletonized/aggregated network models can closely 89 resemble the behaviour of full sized systems under steady state conditions (e.g. Perelman et al. 90 (2008) and Preis et al. (2011)). The second category, parameter uncertainty, refers to the errors of 91 the parameters used to represent system components (e.g. pipe roughnesses, pipe diameters). 92 According to Kang and Lansey (2009), these parameters are time invariant or vary slowly over 93 time. Hence, this source of uncertainty can be neglected for real-time estimation problems. Finally, 
94 measurement/data uncertainty is the uncertainty from measurement devices and, more importantly, uncertainty from the inability to capture the temporal and spatial variation of consumer demands. Because of their high impact on model uncertainty during short periods of time (or in real-time),

97 nodal demands are therefore usually selected as the time varying parameters to be estimated.

98 The issue of short term demand forecasting and real-time demand estimation under uncertainties 99 can be found in some recent studies. Note that the short-term demand forecasting and demand 100 estimation are two different problems. The former focuses on predicting future demands (e.g. 101 Cutore et al. (2008), Hutton and Kapelan (2015) and Alvisi and Franchini (2017)). The latter 102 focuses on estimation of the current demands, which is also the main interest of this paper. This is 103 useful, as demand estimation can be used at regular time steps to verify the accuracy of the 104 predicted value and update the system operations. The problem of near real-time demand 105 estimation has been studied using different approaches. Shang et al. (2006) applied an extended 106 Kalman filter, an iterative linear algorithm for nonlinear state estimation, to approximate water 107 demand patterns. In that paper, water demand patterns were predicted by an ARIMA time series 108 model and were refined using real-time observations. Similarly, Hutton et al. (2012a) introduced 109 a particle filter method and an ensemble Kalman filter for the estimation of a single district meter 110 area, which was assumed to follow a linear time series model. The particle filter model was 111 implemented with and without measurement error to show its effect on the demand prediction 112 uncertainty. An alternative for the demand estimates can be found in Kang and Lansey (2009). In 113 their paper, two comprehensive methods for the demand estimation problem were introduced, the 114 Kalman filter and the tracking state estimator (TSE). For the Kalman filter model, the water 115 demand patterns were also assumed to follow a linear time series model, while the TSE model 116 involved recursively computing the sensitivity matrix (i.e. the Jacobian matrix of the measurement 
117 vector with regards to the change in the state vector). The uncertainties of the demand estimates

118 were suggested to be quantified by applying the first-order second moment formula. The two

119 models were then tested on a case study (116 pipes, 90 nodes, 1 source and 1 tank) with an

120 assumption that 19 flow measurement sites and 5 pressure measurement sites were available. It

121 should be noted that the demand estimation problem is sensitive to the locations and types of the

122 measurements (Do et al. 2016). Demand estimation models usually perform better with flow

123 measurements rather than pressure/head measurements. However, due to the cost and difficulty of

124 installing flow measurement devices compared to pressure measurement devices, flow

125 measurement devices are usually not as commonly used as pressure measurement devices in real

126 WDS networks.

127 In summary, water demands in WDS studies are usually assumed to be known and varied based

128 on a diurnal curve. However, this assumption might lead to large approximations of WDS states

129 in real-time due to the unpredictable variation of the water demands. Some efforts have been

130 focused on the real-time demand estimation. By assuming that the water demand follows a linear

131 time series prediction model, these models approximated the water demand patterns with some

132 linear algorithms such as the Kalman filter or extended Kalman filter. Given the nonlinear

133 stochastic nature of the water demands as well as the need for practical applicability, real-time

134 estimation modeling of WDS still requires much research effort.

135 This paper presents a model framework for the online (near real-time) demand estimation of a

136 WDS, which is named the DMFLive model. A predictor-corrector methodology is adopted in the

137 DMFLive model to predict the hydraulic behaviors of the water network based on a nonlinear

138 demand prediction sub-model, and to correct the prediction by using online pressure observation

139 data. A particle filter method is applied to implement the predictor-corrector approach. The typical 
140 problems of the particle filter approach (particle degeneracy, impoverishment and particle

141 convergence) are investigated by two different resampling schemes: systematic resampling (SR)

142 algorithm and systematic resampling integrated with a genetic algorithm process (SRGA).

143 Uncertainties of model outputs are quantified and evaluated in terms of confidence intervals.

144 The paper is structured as follows. First, an explanation of the state estimation problem and its

145 conceptual solution is introduced. Second, the basic concepts of particle filter methods to solve the 146 estimation problem are explained. This is followed by a detailed description of the particle filter 147 methodology applied for water demand state estimation in WDS. Two case studies are then used 148 to evaluate the model. Finally, conclusions and suggestions for future work are given.

\section{State estimation problem and its conceptual solution}

150 The problem of state estimation involves finding a target state vector $x_{k}$ that evolves according to

151 a discrete time stochastic model (Ristic et al. 2004):

$$
x_{k}=f_{k-1}\left(x_{k-1}, v_{k-1}\right)
$$

152 where $k$ is the index of discrete time steps; $f_{k-1}$ is a known, possibly nonlinear function of the

153 previous state and $v$ is the process noise sequence. The value of $x_{k}$ can be found from

154 measurements $z_{k}$, which are related to $x_{k}$ via the measurement equation:

$$
z_{k}=h_{k}\left(x_{k}, w_{k}\right)
$$

155 where $h$ is a known implicit or explicit, possibly nonlinear function and $w$ is the measurement

156 noise sequence. The noise terms $v_{k}$ and $w_{k}$ are usually assumed to be white noise and independent.

157 From a statistical and probabilistic perspectives, the state model can be represented by a probability 158 density function (pdf). The state estimation problem, therefore, becomes a process of recursively 159 quantifying some degree of belief in the state $x_{k}$ given the measurement series $Z_{k}\left(z_{i}, i=1, \ldots, k\right)$ up 
160 to time $k$. This process can be obtained by two stages: prediction and correction/update. The

161 prediction stage involves applying the system model to predict the prior pdf of the state:

$$
p\left(x_{k} \mid Z_{k-1}\right)=\int p\left(x_{k} \mid x_{k-1}\right) p\left(x_{k-1} \mid Z_{k-1}\right) d x_{k-1}
$$

162 where $p\left(x_{k} \mid x_{k-1}\right)$ is the probabilistic model of the state model, or the transitional probability

163 density function, which is defined by the system equation Eq. (1) with the known statistics of $v_{k-1}$;

164 and $p\left(x_{k-1} \mid Z_{k-1}\right)$ is the pdf of the model at time $k-1$, which is supposed to be known.

165 The correction/update stage implements Bayes' rule to compute the posterior probability density 166 of the state model when the measurement $z_{k}$ becomes available:

$$
p\left(x_{k} \mid Z_{k}\right)=\frac{p\left(z_{k} \mid x_{k}\right) p\left(x_{k} \mid Z_{k-1}\right)}{\int p\left(z_{k} \mid x_{k}\right) p\left(x_{k} \mid Z_{k-1}\right) d x_{k}}
$$

167 where $p\left(z_{k} \mid x_{k}\right)$ is the likelihood function, defined by the measurement equation (Eq. (2)) with the 168 known statistics of $w_{k}$.

169 According to Ristic et al. (2004), the recursive propagation of the posterior pdf shown in Eq. (3)

170 and Eq. (4) is only a conceptual solution that cannot be analytically solved. The solution requires

171 the storage of a fully non-Gaussian pdf, corresponding to an infinitive dimensional vector. Since

172 the true solution is too complex and almost impossible to compute, an implementation of 173 approximation techniques or suboptimal Bayesian algorithms is developed. The following section

174 introduces an approximation technique, namely the particle filter, to solve the aforementioned state

175 estimation problem.

176 Particle filters

177 Over the last decade, particle filters have been successfully applied to the state and parameter 178 estimation of complex system models in various environmental engineering fields, such as 179 hydrology (Moradkhani et al. (2005), Weerts and El Serafy (2006)), hydraulic (Hutton et al. 2012a) 180 and geoscience (van Leeuwen (2010)). Unlike the Kalman filter (for linear problems), extended 
181 Kalman filter (which requires a linearization of the nonlinear problems) or the unscented Kalman 182 filter (which uses a small number of deterministically chosen samples), the particle filter can use 183 a large number of Monte Carlo samples to estimate fully nonlinear, possibly non-Gaussian target 184 states. The key concept of a particle filter is to approximate the posterior pdf of states, defined in 185 Eq. (4), by an ensemble of samples $\left(N_{p}\right)$, each of which contains an associated weight $\left(w_{k}^{i}\right)$, and 186 to compute estimates based on these samples and weights:

$$
\begin{aligned}
& p\left(x_{k} \mid Z_{k}\right) \approx \sum_{i=1}^{N_{P}} w_{k}^{i} \delta\left(x_{k}-x_{k}^{i}\right) \\
& w_{k}^{i}=w_{k-1}^{i} \frac{p\left(z_{k} \mid x_{k}^{i}\right) p\left(x_{k}^{i} \mid x_{k-1}^{i}\right)}{p\left(x_{k}^{i} \mid x_{k-1}^{i}, z_{k}\right)}
\end{aligned}
$$

187 where $\delta$ is the Dirac delta function; $i$ is the particle index; and $p\left(x_{k}^{i} \mid x_{k-1}^{i}, z_{k}\right)$ is the importance 188 density function. In order to simplify the weight update of the particle, the importance density 189 function is usually chosen as the transitional density function, $p\left(x_{k}^{i} \mid x_{k-1}^{i}, z_{k}\right)=$ $190 p\left(x_{k}^{i} \mid x_{k-1}^{i}\right)$, which yields with scaling:

$$
w_{k}^{i}=\frac{p\left(z_{k} \mid x_{k}^{i}\right)}{\sum_{i=1}^{N_{P}} p\left(z_{k} \mid x_{k}^{i}\right)}
$$

191 These equations form the basis of most particle filters. However, it has been shown by Doucet et 192 al. (2000) that the variance of the weights will increase over time if the particle filtering process is 193 limited at executing only these equations. Since the particles drift away from the "truth" as well as 194 obtain negligible weights (Moradkhani et al. 2005), the model will fail to estimate the real states 195 of the system. To avoid this problem, a resampling process, which replaces samples with low 196 importance weights by the samples with high importance weights, is added to the procedure of 197 particle filter models. In this paper, the systematic resampling method, also called the stochastic 198 universal resampling, introduced by Kitagawa (1996), is selected for the resampling procedure of 199 the particle filter model. A comprehensive explanation of the systematic resampling and the full 
200 review of particle filtering methods are described in (van Leeuwen 2009). In addition, an improved 201 resampling method which integrates the evolutionary scheme from genetic algorithms into the

202 resampling process, is also proposed to improve the efficiency of the particle filter model.

\section{Particle filters applied for water demand state estimation in WDS}

204 In this study, the predictor-corrector approach implemented by a particle filter model for the

205 estimation of water demands in real-time is proposed, namely the DMFLive model. The demand 206 prediction sub-model presented by van Zyl et al. (2008) has been applied to predict the water 207 demand multipliers (DMF) in a WDS. The hydraulic EPANET toolkit (Rossman 2000) which 208 solves the hydraulic equations was used to compute the model equivalent of the measurement data

209 (i.e. the nodal pressures, flow rates at measurement locations or the final tank levels at the end of 210 each time step). These computed values then were integrated with the corresponding field 211 measurements in order to correct/update the particle weights. Particles were, thereafter, resampled 212 (with either SR or SRGA) and subsequently used as input for the prediction model. 213 Simultaneously, the estimated demand multipliers were computed and selected for uncertainty 214 quantification. The uncertainties of the demand multipliers caused by the errors from measurement 215 devices were computed using the first-order approximation formula. The flowchart of the 216 DMFLive model is shown in Figure 1.

\section{Initialization of particles}

218 The DMFLive model starts with a creation of an ensemble of the particles $\left(N_{p}\right)$. The particles are 219 the demand residuals, driven by the demand prediction model to predict the demand multipliers. 220 In addition, each particle is assigned an initial weight equal to $1 / N_{p}$.

\section{Demand prediction sub-model}


222 The initial particles (for the first iteration) or the particles after resampling (from the second 223 iteration onwards) are transferred to the demand prediction sub-model. Demand residual 224 information carried by the particles is used to track the states and predict the demand multipliers 225 via the following equations (van Zyl et al. 2008):

$$
\ln x_{k}^{j}=\sum_{i=1}^{m} \phi_{i}^{j} \ln x_{k-i}^{j}+\ln v_{k}^{j}
$$

226 where $x_{k}^{j}$ is the demand residual state at time step $k$ of the $j^{\text {th }} \mathrm{DMF} ; i$ is the lag counter; $m$ is the 227 number of autocorrelation lags (for the state estimation problem $m=1$ as referred to Eq. (1)); $\phi_{i}$ is 228 the auto-regression coefficient for lag $i$ and $v_{k}\left(0, \sigma_{\mathrm{h}}\right)$ is the white noise with mean zero and standard 229 deviation $\sigma_{h}$.

230 The $j^{\text {th }}$ DMF is calculated as:

$$
D M F_{k}^{j}=C_{k}^{j} x_{k}^{j}
$$

231 where $C_{k}^{j}$ is the value at time $\mathrm{k}$ of a typical diurnal demand pattern of the $j^{\text {th }} \mathrm{DMF}$. The $\mathrm{C}$ value 232 can be identified based on meter information of different water users (e.g. in Beal and Stewart 233 (2014)).

\section{Real-time hydraulic data}

235 In practice, hydraulic data can be captured in real-time via the SCADA system or sensor devices.

236 For the DMFLive model, two types of real-time hydraulic data are required. First are the tank

237 levels, pump and valve statuses, and second are the nodal heads and pipe flow rates at measurement

238 locations. Tank levels, pump and valve statuses are used as boundary conditions for the hydraulic

239 simulation of the water network model while the observations at measurement locations are used 240 to correct/update the weight of the particles.

241 In order to validate the performance of the proposed model as well as its practical applicability to 242 real WDS networks, all case studies in this research are assumed to have pressure measurements 
243 only. The input data sets to evaluate the DMFLive model are synthetically generated based on

244 deterministic models, where the water network parameters are fully known, as follows: (1) known

245 demand patterns are assigned to nodal demands; (2) EPANET is run to record tank levels, pump

246 statuses, and pressures at selected measurement locations; (3) to introduce the measurement errors,

247 a normal distributed random error in an allowable range $\left( \pm \Delta^{\text {meas }}\right)$ is added to each nodal pressure.

$248 \quad$ Simulator

249 The hydraulic behavior of the water distribution network at each time step is simulated using an

250 EPANET steady state simulation. The inputs are the predicted DMFs, tank levels, and pump and

251 valve statuses. The water network characteristics such as pipe lengths, diameters, roughness

252 coefficients, node elevations, pump curves, etc. are assumed to be known and constant. The outputs

253 from the EPANET hydraulic solver is the model equivalent of the observations, i.e. the simulated

254 nodal heads and pipe flow rates at measurement locations.

\section{Corrector}

256 The weights of the particles are corrected/updated by associating the simulated heads and flows

257 with the actual observations via Eq. (7) where the likelihood function is assumed to be Gaussian:

$$
p\left(z_{k} \mid x_{k}^{i}\right)=\frac{1}{\sqrt{2 \pi|R|}} e^{\left(-\frac{1}{2}\left[z_{k}-h\left(x_{k}^{i}\right)\right]^{T} R^{-1}\left[z_{k}-h\left(x_{k}^{i}\right)\right]\right)}
$$

258 where $h\left(x_{k}^{i}\right)$ is the model equivalent of the observations $z_{k}$ (simulated nodal heads and flow rates),

259 and $\mathrm{R}$ is the covariance matrix of the observation errors, which in general is caused by errors from

260 two main sources: forward model error and measurement device error. The forward model error,

$$
\Delta^{\text {true }}=Z^{\text {true }}-h\left(x^{\text {true }}\right)
$$

261 is the difference between the true observation vector, $Z^{\text {true }}$, and the corresponding vector output

262 from the hydraulic simulation model EPANET using the true state $x^{\text {true }}$. The true observation vector 
263 is a theoretical vector that represents observations measured by perfect measurement devices. It

264 is linked to the actual measured values via the expression:

$$
Z=Z^{\text {true }}+\Delta^{\text {meas }}
$$

265 The observation error covariance matrix, therefore, can be estimated as $R=R^{\text {true }}+R^{\text {meas }}$, where

$266 R^{\text {true }}$ and $R^{\text {meas }}$ denote the covariance of the forward model error and the covariance of measurement

267 error, respectively (see Waller (2013) for a detailed explanation and calculation of the observation

268 error covariance matrix). To produce good estimates of the model state in real case studies, the

269 error covariance matrix must be well understood and properly calibrated. As previously mentioned

270 in this paper, the measured data in all case studies were synthetically generated from the EPANET

271 model based on "true" demand patterns. The forward model error, therefore, equals to zero. The

272 covariance matrix $\mathrm{R}$, as a result, is the diagonal matrix where the diagonal elements are the

273 variances of the measurement errors, since observations are independently measured at different

274 locations of the network by different measurement devices. The measurement errors with specified

275 ranges are assumed to be known so that the covariance matrix R can be identified. Resampling

276 Resampling is applied to create new ensembles of particles from the posterior pdf of the previous

277 step. In this paper, two alternatives of resampling are tested: systematic resampling algorithm (SR)

278 and systematic resampling integrated with the GA operators (SRGA).

279 The SR algorithm generates a random number $u_{s}$ from the uniform density $\mathrm{U}[0,1 / N p]$, and 280 consequently creates $N_{p}$ ordered numbers (Hol et al. 2006):

$$
u^{i}=\frac{i-1}{N_{P}}+u_{s} \quad\left(i=1, \ldots, N_{P}\right)
$$

281 New particles are then selected that satisfy Eq. (14):

$$
x_{\text {new }}^{i}=x\left(F^{-1}\left(u^{i}\right)\right)
$$


282 where $F^{-1}$ denotes the generalized inverse of the cumulative probability distribution of the 283 normalized particle weights.

284 To reduce the convergence problem of the particles (i.e. all the particle weights are equal to zero)

285 when applying the model for large networks with multiple demand patterns, the SRGA method is

286 also applied. Three GA operators of selection, crossover and mutation are responsible for 287 modifying the predicted demands before computing the weight of a particle by Eq. (10). In the 288 selection step, particles are compared to each other through tournament selection and the best 289 particles are selected as parents. Parent particles are then paired and go through crossover and 290 mutation to generate offspring solutions. While the details of GA can be found in Nicklow et al. 291 (2010), it is important to know that new parameters need to be introduced: the probability of 292 crossover $P_{c}$, the probability of mutation $P_{m}$ and the number of generations $N_{g e n}$.

293 Demand multiplier outputs and uncertainty quantification

294 The estimate of the state $x_{k}$ is obtained by taking the mean of the particle filter sample set (Salmond 295 \& Gordon 2005):

$$
\hat{x}_{k} \approx \frac{1}{N_{p}} \sum_{i=1}^{N_{p}} x_{k}^{i *}
$$

296 where $x_{k}^{i *}$ is the state updated based on the posterior analysis of the model weights.

297 For particle filter models, the uncertainty of the model output can be computed by taking the 298 variance of the samples:

$$
\operatorname{var}\left(x_{k}\right) \approx \frac{1}{N_{p}} \sum_{i=1}^{N_{p}}\left(x_{k}^{i *}-\hat{x}_{k}\right)\left(x_{k}^{i *}-\hat{x}_{k}\right)^{T}
$$

299 For the demand multiplier estimation problem, it should be noted that a small change in the demand

300 multiplier can cause a large change in nodal demands (for nodes with large base demands) and 301 consequently result in large variations of nodal pressures, especially at nodes that are sensitive to 
nodal demands. Most of the demand forecasting models are required to capture both peak-demand hours and off-peak demand hours, with a demand multiplier factor that can vary from 0 to 4 (Chin et al 2000). The weight of the particles via Eq. (10) can, therefore, easily approach zero which leads to either particle degeneracy or particle non-convergence. Using a larger number of particles can prevent this problem, however, if the dimension of the state vector increases, the required number of particles increases exponentially. One way to solve these issues is to incorporate the covariance of the forecasting nodal heads/ pipe flow rates into the likelihood function:

$$
p\left(z_{k} \mid x_{k}^{i}\right)=\frac{1}{\sqrt{2 \pi\left|R^{*}\right|}} e^{\left(-\frac{1}{2}\left[z_{k}-h\left(x_{k}^{i}\right)\right]^{T}(R *)^{-1}\left[z_{k}-h\left(x_{k}^{i}\right)\right]\right)}
$$

309 where $\mathrm{R}^{*}=\mathrm{R}+\Sigma, \Sigma$ is the covariance matrix of the forecast nodal heads or pipe flow rates, 310 computed based on the forecast demands. This covariance matrix can be estimated by running the 311 demand forecasting model multiple times to obtain the range of forecast demand multipliers, then 312 applying these values into the hydraulic model to compute the variance of simulated nodal heads 313 and pipe flow rates at measurement locations.

314 Although the method can ensure some of the particles always contain weights to avoid particle 315 non-convergence and degeneracy, this would increase the noise of the output model. The variance 316 of the model output (i.e. the uncertainty of the model output) is required to be computed by a 317 different method instead of using Eq. (16).

318 Another way to overcome the convergence and degeneracy issues is to integrate the GA operators 319 into the resampling process as mentioned in the previous sections. The integrated GA approach 320 can prevent the model from experiencing these problems by exploring the state-space region and 321 selecting the best particles (including the replication of good solutions). However, it might lead to 322 another problem for the particle filter, referred to as particle impoverishment. The distribution of 
323 the state model, because of the particle impoverishment, is poorly represented by only one or a

324 few particles which significantly reduces the variance of the model state.

325 To ensure reliable outputs from the particle filter model, it is proposed to approximate the uncertainty of the model state by an independent method, such as the first-order approximation (FOA) method adopted from Piller (1995). This also has the advantage of significantly decreasing

328 the computational time, as it will be shown in the case studies. The model outputs, therefore, are 329 the estimate of the demand multipliers computed by Eq. (15) and the confidence intervals 330 computed by FOA method. For example, the 95\% confidence interval of the estimated demand 331 multiplier (i.e. the range in which the true demand multipliers are expected to be $95 \%$ of the time)

332 can be obtained by the following expression:

$$
\begin{gathered}
\left\|\Delta D M F_{k}\right\| \leq 1.96\left(W^{\frac{1}{2}} J\right)^{\dagger} \\
\left|\Delta D M F_{k}^{j}\right| \leq 1.96 \sum_{j=1}^{m}\left|S_{i j}\right|, \text { with } S=\left(W^{\frac{1}{2}} J\right)^{\dagger}
\end{gathered}
$$

333 where $J$ is the Jacobian matrix of flows and heads with respect to the water nodal demand at time

$334 k ; W$ is the weight matrix where the diagonal elements are the reciprocals of the variances of 335 measurement errors $\left(W=R^{-1}\right)$; superscript $\uparrow$ represents the pseudo-inverse operator. The derivation 336 of Eq. (18) is explained in detail in Appendix A.

337 By considering the Jacobian (sensitivity) matrix, the uncertainty of the output model from FOA 338 method can provide meaningful information about the sensitivity of the pressure with respect to 339 the change in the nodal demand. This information can be used to guide where to place 340 measurement stations. However, the method requires calculation of the sensitivity matrix, which 341 may be time consuming when applied to large and complex networks.

\section{Summary of assumptions and input requirements for the DMFLive model}

343 Several assumptions are made for this study: (1) the model of the water distribution network 344 perfectly represents the real system with known network characteristics (e.g. pipe roughness 
345 coefficients, length and diameters, etc.), and only demand multipliers are required to be estimated;

346 (2) typical demand patterns for different homogeneous demand groups in WDS are assumed to be

347 known. The homogeneous demand groups can be identified based on a multi-criteria demand

348 zones clustering algorithm presented in Preis et al. (2010). There is uncertainty of the model

349 outputs associated with demand groupings, but this is not considered here. Therefore, (3) the

350 source of uncertainty is only from the errors from measurement devices; (4) the errors of the

351 measurement devices are assumed to be known and to follow a Gaussian distribution; (5) the

352 observation data for the online (near real-time) estimation model is available every 10, 15 minutes,

3531 hour or larger time steps. The influence of slow transients (mass oscillations) are, therefore,

354 ignored in this context.

355 The inputs required for the DMFLive model consist of the number of particles, the inputs for the 356 demand prediction sub-model, inputs for the hydraulic simulation model (EPANET), input for the 357 correction step and the parameters for the integrated GA operators $\left(P_{c}, P_{m}\right.$ and $\left.N_{g e n}\right)$. The prediction 358 sub-model requires the data of typical demand patterns, the auto-regression coefficient $\left(\phi_{i}\right)$ and the 359 variance of noise of demand residuals $\left(\sigma_{h}^{2}\right)$. These parameters are calibrated independently based 360 on historical demand data for specific networks, for example $\phi_{I}=0.7$ and $\sigma_{h}^{2}=0.13^{2}$ as in van 361 Zyl et al. (2008). The EPANET model requires the known data of tank levels, pump and valve 362 statuses. The correction step requires the observation data at measurement sites. Note that the 363 particle filter model associated with the GA process can only be applied to networks with multiple 364 demand patterns (e.g. the second case study in this paper). Two-point crossover operator with the 365 probability of crossover $P_{c}=0.7$, bitwise mutation with the probability of $P_{m}=1 / N_{D M}\left(N_{D M}\right.$ is the 366 number of demand patterns in the network, $N_{D M}=5$, corresponding with $P_{m}=0.2$ for the second 367 case study) and the number of generations $N_{G e n}=50$ were selected for the GA process. 


\section{Case study 1}

369 The first case study used to evaluate the model is shown in Figure 2. The network has 9 nodes $(8$ 370 nodes with demands), 12 pipes, one tank and one reservoir. The network characteristics can be

371 found from the EPANET example, namely the Netl network. Three pressure measurements (with 372 a precision of $\Delta^{\text {meas }}= \pm 0.2 \mathrm{~m}$, consistent with a standard deviation of $\sigma^{\text {meas }}=0.1$ for the 373 measurement error at 95\% confidence interval) are assumed to be placed at three random locations

374 (nodes 13, 22 and 31). All nodal demands are assumed to follow a single demand pattern that 375 varies every 15 minutes, (represented by the continuous line in Figure 2.b). The demand pattern is 376 a random daily demand pattern (from a yearly demand pattern) for 100 households obtained from 377 the BESS model (Thyer et al. 2011). The DMFLive model is required to track this demand pattern 378 using the three pressure measurements, which are also obtained every 15 minutes.

379 In this case study, the default demand pattern given in the Net1 example (represented by the dashed 380 line in Figure 2.b) was selected as the typical demand pattern. Different values of the auto381 regression coefficient $(\phi)$ as well as variance of noise $\left(\sigma_{h}^{2}\right)$ were applied for the demand prediction 382 sub-model.

383 The accuracy of the demand estimates from the DMFLive model were evaluated in terms of the 384 coefficient of determination $\left(R^{2}\right)$ and the root mean squared error (RMSE). For a number of 385 particles $N_{p}=100$, the results of the demand estimates from the DMFLive model are presented in 386 Table 1.

387 The DMFLive model performed very well when the auto-regression coefficient was selected in the 388 range of $0.3 \leq \phi \leq 0.9$ and the noise variance was selected in the range of $0.25 \leq \sigma_{h}^{2}<0.64$. Due 389 to the large difference between the typical demand value and the actual demand value at each time 390 step (Figure 2.b), the selection of small values of the auto-regression coefficient and noise variance 
391 resulted in relatively poorer performance of the model (e.g. $\mathrm{R}^{2}=0.465$ and $\mathrm{RMSE}=0.198$ for $392 \phi=0.3$ and $\left.\sigma_{h}^{2}=0.04\right)$. The best output of the DMFLive model was obtained at $\phi=0.7$ and $\sigma_{h}{ }^{2}=0.25$, 393 with $\mathrm{R}^{2}=0.988$ and $\mathrm{RMSE}=0.028$, respectively.

394 For this best estimated demand pattern, the confidence intervals and the scattergram between 395 actual demand multipliers and estimated demand multipliers are plotted in Figure 3.a.

396 In Figure 3.a, the estimated demand pattern yields a very good match with the actual demand 397 pattern during the time period ( 24 hours, corresponding to 96 time steps). The actual demand 398 pattern is entirely covered by the range of the $95 \%$ confidence intervals calculated from FOA 399 method. This confidence interval range, which is expected to bracket the "true" demand multipliers 400 in $95 \%$ of the cases, represents the uncertainty magnitude of the estimated demand due to the error 401 from measurement devices.

402 The model has also been run with the number of particles $N_{p}=100$ and $N_{p}=20$ to provide a 403 comparison between the FOA method (i.e. Eq. (18)) and the posterior analysis (i.e. Eq. (16)) for 404 uncertainty quantification, as shown in Figures 3.b, 3.c and 3.d. Figures 3.a and 3.c show the 405 uncertainty quantified by the FOA method while Figures 3.b and 3.d shown the uncertainty 406 quantified by the variance of particles. For $N_{p}=100$ particles, the $95 \%$ confidence intervals from 407 both methods are comparable to each other, which demonstrates that the FOA method can provide 408 reliable results compared to the variance of the particle filter samples.

409 A good estimate of the demand multipliers $(R M S E=0.047)$ is obtained by the DMFLive model 410 even when the number of particles is reduced by a factor of five $\left(N_{p}=20\right)$, as seen in Figures 3.c, 411 and 3.d. The uncertainty boundary calculated by the FOA method in Figure 3.c has a similar range 412 to the case with $N_{P}=100$ particles and covers most of the actual values. On the other hand, the 413 uncertainty bounds calculated by Eq. (16) in Figure 3.d are collapsed into single value at some 
414 time steps due to an insufficient number of the particles. Application of Eq. (16) for uncertainty

415 quantification, therefore, requires an in-depth evaluation of the number of particles in the model if

416 it is selected for the uncertainty quantification.

417 The range of demand multipliers predicted in time according to the evolution of the particles is 418 presented in Figure 4.a. The predicted values range from $D M F_{\min }=0.1$ to $D M F \max =7.0$,

419 indicating that the demand prediction sub-model can predict a large range of demand multipliers, 420 and cover the range $0 \leq D M F \leq 4$ suggested by Chin et al. (2000). Figure 4.b plots the scattergrams 421 of the actual demand multipliers versus the predicted demand multipliers (i.e. the mean of the 422 prediction) and actual demand multipliers versus estimated demand multipliers. The scattergram 423 shows a constant and strong correlation between actual demand multipliers and estimated demand 424 multipliers over time with $\mathrm{R}^{2}$ being close to unity. Due to large difference between the typical 425 demand pattern and the actual demand pattern, the forecasting model does not provide good 426 prediction, resulting in weak and skewed correlation between the actual values and the predicted 427 values. Despite this, the DMFLive model is still capable to provide very good estimates of the 428 demand multipliers.

\section{$429 \quad$ Effects of tank level update on the estimation}

430 In extended period simulations of most hydraulic solvers (including EPANET), the nodal demands 431 are considered to be constant during the time step. The levels of the tanks in the network at the end 432 of the time step are consequently computed based on this assumption and are used as the initial 433 tank level for the next step. Due to continuously unpredictable change of the water demand in 434 practice, the actual tank level at the end of the time step is usually different to the tank level 435 computed by the model. As a result, the estimated total volume of water used during the time step 436 is also different from the actual volume of water used in practice. This issue can be overcome by 
437 minimizing the difference between actual tank levels at the beginning of the time step and the final 438 estimated tank level at the end of the previous step. The demand estimation model, however, will 439 be delayed until the information of the tank level at the beginning of the next time step becomes 440 available. In other words, the model outputs will be the estimates of the demand multiplier at the 441 previous time step.

442 In order to evaluate the effect of including tank level information at the end of every time step, an 443 additional test is conducted. Instead of assuming that the observations are available at every 15 444 minutes, in this test it is assumed that the data can be obtained every hour and the model is required 445 to estimate the demand pattern at each hour time step (while the actual demand pattern is varied 446 every 15 minutes).

447 Figure 5 plots the two estimated demand patterns with and without tank level information (herein 448 referred to as DMF-WTLive and DMFLive). Note that the DMF-WTLive model is the modified 449 version of DMFLive model at which the final tank level information is taken into account.

450 It can be seen that the estimates for both cases are matched with the actual demand pattern at every 451 hour time step. The inclusion of tank information only causes a slight difference between two 452 estimated demand patterns at some of the time steps. The root mean squared errors between 453 estimated demand multipliers and actual demand multipliers at every hour step indicates that the 454 DMFLive model obtained slight better results than the DMF-WTLive model (RMSE $=0.046$ 455 compared to RMSE $=0.080$, respectively). However, the total estimated water usages tabulated in 456 Table 2 shows that the DMF-WTLive model is more accurate in predicting the volume of water 457 delivered to the users.

458 The total estimated water usage during the 24-hour simulation period from DMFLive model was $4595942.43 \mathrm{~m}^{3} /$ day, $46.81 \mathrm{~m}^{3} /$ day (or $0.78 \%$ ) less than the actual water usage. On the other hand, total 
460 estimated water usage from $D M F$-WTLive model was $6007.31 \mathrm{~m}^{3} /$ day, only $18.07 \mathrm{~m}^{3} /$ day (or $461 \quad 0.30 \%$ ) more than the actual value. Therefore, if the estimation can be delayed one time step, the

462 final tank level information should be included into the model to improve the accuracy of the 463 estimated total volume of water used.

\section{Case study 2}

465 In order to evaluate the performance of the proposed model in large networks that contain more 466 than one demand pattern, the C-Town network from Ostfeld et al. (2011) is selected as the second 467 case study. The network consists of 429 pipes, 1 reservoir, 7 tanks, 5 pump stations (with a total 468 of 11 pumps), 4 PRV valves and 388 nodes (334 nodes with demands), which are divided into five 469 district demand areas. Each district demand area follows a different hourly demand pattern. As the 470 data of the demand patterns is available for seven days, the first 24 hours of these demand patterns

471 are assumed to be the typical demand patterns for the demand prediction sub-model. The 472 performance of the particle filter model is then evaluated by estimating the remaining 6-day hourly 473 demand patterns.

474 It is assumed that there are 14 pressure measurement sites (from P1 to P14) that are randomly 475 located at 14 places. These pressure measurements, again, are assumed to have a measurement 476 error of $\Delta^{\text {meas }}= \pm 0.2 \mathrm{~m}$. The inputs for the real-time demand estimation model are, therefore, the 477 pressures at these locations, the tank levels of seven tanks and the pump statuses of 11 pumps at 478 each hour time step. The topology and measurement locations of the C-Town network are shown 479 in Figure 6. Five different demand prediction sub-models were used to predict the five demand 480 patterns. The parameters of the five demand prediction sub-models were assumed to have the same 481 values of $\phi=0.7$ for the auto-regression coefficients and $\sigma_{h}^{2}=0.16$ for the variances of noise. 
482 The standard particle filter model (i.e. using systematic resampling), herein referred as the 483 DMFLive-I model, provides good results only if $N_{P} \geq 25,000$ particles. The estimates of five 484 different demand patterns for 6 days (from $25 \mathrm{~h}$ to $168 \mathrm{~h}$ ) are shown in Figure 7 . It is seen that the 485 estimated demand patterns closely match the actual demand patterns, especially for DMF 2 (RMSE $486=0.021), \mathrm{DMF} 3(\mathrm{RMSE}=0.024), \mathrm{DMF} 1(\mathrm{RMSE}=0.029)$ and DMF $4(\mathrm{RMSE}=0.036)$. The 487 estimated demand pattern DMF 5 is less accurate, with the root mean squared error of RMSE $=$ $488 \quad 0.061$.

489 Figure 7 also plots the 95\% confidence intervals for calculated by the FOA formula. The intervals 490 for the estimated DMF 1, DMF 2 and DMF 3 (in Figure 7.a, 7.b and 7.c, respectively) are narrow 491 and they cover almost the entire set of the actual demand multiplier values. The actual values of 492 DMF 4 are also within the confidence interval of estimated DMF 4 (Figure 7.d) for most of the 493 time. However, due to the locations of the measurements (P7 and P9 - Figure 6), the confidence 494 interval of estimated DMF 4 pattern is relatively large compared to the others. The effect of 495 measurement locations on the confidence intervals of the estimates is discussed later in the paper. 496 In Figure 7.e, approximately $37 \%$ of the actual demand values of the demand pattern DMF 5 are 497 outside the $95 \%$ confidence intervals, which is caused by the relatively poor estimates for DMF 5. 498 Figure 8 displays the scattergrams and coefficients of determination of the five predicted demand 499 patterns, as well as the estimated demand patterns versus their actual values.

500 The predicted DMFs in this case show an average correlation to the actual DMFs with the $\mathrm{R}^{2}$ 501 ranging from 0.69 to 0.74 , while the estimated DMFs are strongly correlated to the actual ones

502 with all $\mathrm{R}^{2}$ values being close to unity. The estimation for these five DMFs are also reliable during 503 the simulation period (six days), as the spreads of the scattered dots are close to bisector lines.

\section{Improving DMFLive model performance by SRGA and modified likelihood function}


505 The DMFLive-I model can only perform well with a large number of particles $\left(N_{P} \geq 25,000\right)$.

506 Smaller numbers of particles result in weak estimates of the DMFs due to particle collapse at some

507 steps. Since increasing the number of demand patterns requires an exponentially increasing 508 number of particles, it is necessary to improve the efficiency of particle filter model so that it can 509 be applied to complex systems.

510 Two methods have been investigated as mentioned previously in the paper: (1) incorporating the 511 variance of the forecasting nodal heads into the likelihood function. The weights of particles in the 512 model, referred as DMFLive-II model, are then calculated by the modified likelihood function (Eq. 513 (17)); and (2) by the integration of a GA process into the systematic resampling of the model, 514 herein referred as DMFLive-III model.

515 Table 3 presents results (in terms of the RMSE of each demand pattern) of running these models 516 with $N_{P}=1000$ and $N_{P}=5000$ for DMFLive-I, II and with $N_{P}^{G A}=20$ and $N_{P}^{G A}=100$ for DMFLive$517 I I I$. It may be seen that for both $N_{P}$ values, the DMFLive-I gives very poor estimates of the DMFs. 518 On the other hand, the DMFLive-II model only requires $N_{P}=1000$ (corresponding to $1.43^{*} 10^{5}$ 519 evaluations for 143 hours) to provide fairly good results, while the DMFLive-III performs well 520 when $N_{P}^{G A}=100$. The results of DMFLive-II $\left(N_{P}=5000\right)$ and DMFLive-III $\left(N_{P}^{G A}=100\right)$ give 521 similar to the results of DMFLive-I running at $N_{P}=25,000$ (corresponding to total evaluations of $5223.575^{*} 10^{6}$ ). This means the computation can be reduced by approximately a factor of five times.

523 Figure 9 shows the DMF 1 uncertainty ranges from 25 to 49 hours of the three models DMFLive $524 I, I I$ and $I I I$ computed by FOA method and by variance of the particles Eq. (16). As can be seen 525 from Figures 9.a and c, due to particle impoverishment, the uncertainty computed by particle 526 variance, represented by the dashed lines, is merged into a single line at almost all of the time 
steps. The uncertainty in Figure 9.b computed by this method is wide due to the incorporation of the forecasting nodal heads into the likelihood function.

On the other hand, the uncertainties by FOA method, which are directly computed from the sensitivity matrix and the measurement errors, show consistent ranges in both cases. Given good estimates of the demand multipliers (as in Figures 9.b and c) these ranges can cover the actual 533 values most of the time.

\section{Effect of the locations of measurements on the quantification of demand uncertainty}

535 As discussed in a number of studies such as in Piller (1995) and Do et al. (2016), the locations of the measurements have a strong impact on the results of the demand estimation models.

537 Furthermore, the selection of measurement locations also affects the confidence intervals of the estimation outputs.

539 From the mathematical point of view, the uncertainty of estimated demands depends on the

540 sensitivity of the flows/heads at measurement locations in relation to the change in the water nodal

541 demands. This sensitivity is represented by the sensitivity matrix $J$ (Eq. (18)), which is, in this case

542 study, the Jacobian matrix of the heads with respect to the demand multipliers. The sensitivity of

543 the heads with respect to the change of the demand multipliers depends on two factors: (1) the

544 position of the nodes in the network and (2) the base demands at the nodes. In fact, the nodes close

545 to fixed-head nodes (tanks or reservoirs) are less sensitive than the ones far from the fixed-head 546 nodes. This is because of a change in nodal demands will result in a smaller change in the pressures 547 of the closer nodes than the farther nodes. In a similar way, small base demands in the same pattern 548 will result in small friction losses and consequently small changes in pressures. Therefore, nodes 549 selected in these regions may cause large uncertainty in demand multiplier estimation. The 550 sensitivity matrix takes into account these two factors. Small values in the sensitivity matrix values 
551 mean that the nodes are less sensitive to the demands and the estimation might have large 552 uncertainty. Therefore, the uncertainty of the estimated DMFs can be reduced by selecting the 553 more sensitive locations in the network.

554 Let us conduct an additional test to evaluate the effect of the measurement locations on the 555 uncertainty of the estimated demand multipliers, for example the uncertainty of the estimated DMF 556 4. For this test, the locations of measurements P7 (with the base demand of $D_{7}^{0}=0.50 \mathrm{~L} / \mathrm{s}$ ) and P9 $557\left(D_{9}^{0}=0.59 \mathrm{~L} / \mathrm{s}\right)$ are relocated to P7A $\left(D_{7 A}^{0}=1.33 \mathrm{~L} / \mathrm{s}\right)$ and P9A $\left(D_{9 A}^{0}=1.13 \mathrm{~L} / \mathrm{s}\right)$. The DMFLive 558 model was implemented with the same conditions and the other measurement locations are fixed 559 at the same places as the original test.

560 Figure 10 shows the sensitivity matrixes $J_{0}$ (for the original test) and $J_{0}^{A}$ (for the modified test) 561 corresponding to a set of estimated values DMFs $=[0.46 ; 0.54 ; 0.65 ; 0.47 ; 0.62]$

562 It is seen that, for this network, the heads at measurement locations are only sensitive to the change 563 of the DMF that they belong to. For example, the variation in the DMF 4 pattern only affects the 564 sensitivity of the heads at measurement locations P7 and P9 (for original test) and at measurement 565 locations P7A and P9A (for the modified test). The non-zero values in the sensitivity matrices, 566 therefore, correspond to the measurement locations. For the sensitivity of the heads, the new 567 locations P7A $\left(\frac{\partial H}{\partial D M F 4}=5.31\right)$ and P9A $\left(\frac{\partial H}{\partial D M F 4}=11.76\right)$ are considerably more sensitive than the 568 locations $\mathrm{P} 7\left(\frac{\partial H}{\partial D M F 4}=2.59\right)$ and $\mathrm{P} 9\left(\frac{\partial H}{\partial D M F 4}=2.55\right)$. As a result, the confidence intervals of the 569 estimated DMF 4 for the modified test, as shown in Figure 11, are much narrower than the 570 confidence intervals of the estimated DMF 4 for the original test presented in Figure 7.d. Note that 571 in this network case study, the demand patterns are well geographically distributed. The heads at 572 measurement locations are, therefore, affected by independent demand patterns, which results in a 573 narrow uncertainty range for the estimate. For non-geographically distributed DMF networks, the 
574 sensitivity of the heads at measurement locations are required to be accounted and accumulated

575 for all the related DMFs. This might cause much larger uncertainty and likewise bring difficulty

576 for the estimation of the demand multipliers, as has been addressed in Sanz and Pérez (2014).

577 The relocation of the pressure measurements also improves the estimation of DMF 4, with a RMSE

$578=0.028$ for the modified test, compared to a $\mathrm{RMSE}=0.036$ of the original test. The placement of

579 the two new measurement sites also causes a slight difference in the results of other estimated

580 DMFs due to the change in the particle weights. However, the results of the four remaining DMFs

581 are still very good and similar to the estimated values of the original test.

582 To sum up, the uncertainty of estimated demand multipliers caused by the errors of measurement

583 devices is influenced by the measurement locations. It is suggested to choose the locations that are

584 more sensitive to the demand multipliers (see Do et al. (2016) for an example of optimal

585 measurement location). However, it has also been shown that the DMFLive model can be used to

586 estimate the demand multipliers even when the measurement devices are located at some less

587 sensitive places. The uncertainty of the estimated demand multipliers can be used to identify which

588 measurement locations need to be improved. This is another advantage of the DMFLive model.

\section{Conclusions and recommendations}

590 Real-time demand estimation under uncertainties is exceptionally difficult due to the unpredictable

591 stochastic behavior of the water demand as well as the nonlinearities of hydraulic systems. In this

592 paper, the DMFLive model framework has been introduced, which can be used to estimate the

593 demand multipliers of a WDS in near real-time. A predictor-corrector approach has been adopted

594 and solved by a particle filter method. A nonlinear demand prediction model is applied to predict

595 water demand multipliers at each time step, while the online pressure observations are used to

596 correct the prediction. Output uncertainty caused by the measurement errors has also been 
quantified by the first-order approximation formula. The performance of the DMFLive model is evaluated by two WDS case studies. The results showed that the nonlinear demand prediction model combined with the particle filter method used in the paper are well suited for the near realtime demand estimation problem.

601 Within the first case study, the benefits of having additional information about the tank level of

602 the next time step have been explored. If the estimation of the demand multipliers can be delayed 603 one time step, the tank level at the beginning of the next time step can be used by the model to 604 improve the estimation of the total volume of water used.

605 Within the second case study, three versions of the DMFLive model were developed to be used in

606 large networks with multiple demand patterns. All versions provided good results, showing that 607 the models are capable to be used in large networks. Finally, the effect of the measurement 608 locations on the uncertainty of the estimated demand multipliers has been explored. Results 609 showed that the uncertainty can be used to identify which measurement locations need to be 610 improved. Future work involves considering adding additional uncertainties into the DMFLive 611 model. Moreover, testing the model for non-geographically distributed demand networks is also 612 necessary to show its capability when applied in practice.

\section{Appendix}

615 The problem of demand calibration involves finding the demands of the network hydraulic model 616 to best fit the data set. Consider the nonlinear regression equation:

$$
y_{i}^{\text {Meas }}=y_{i}(x)+\varepsilon_{i}, \quad \varepsilon_{i} \sim N\left(0, \sigma_{i}\right)
$$

617 where $x$ is the $n_{d}$ by 1 vector of parameters to calibrate (the demand multiplier factors that depend 618 on time); $y_{i}(x)$ is the scalar multivariate function of predictions from the network hydraulic model, 619 given the parameter $x$; $\varepsilon_{i}$ is the residual between model prediction and observation, which is 
620 assumed to be Gaussian with mean of zero and standard deviation of $\sigma_{i} ; y_{i}^{\text {Meas }}$ is the $i^{\text {th }}$

621 measurement site in the data set.

622 The demand calibration can be formulated as a box-constrained Least Squares problem that 623 minimizes the differentiable criterion at each time step:

$$
\begin{gathered}
f(x)=\frac{1}{2} \sum_{i=1}^{m}\left(\frac{y_{i}(x)-y_{i}^{\text {Meas }}}{\sigma_{i}}\right)^{2}=\frac{1}{2} \sum_{i=1}^{m} \varepsilon_{R}^{2} \\
\text { s.t } x^{\text {min }} \leq x \leq x^{\text {max }}
\end{gathered}
$$

624 where $\mathrm{m}$ is the number of measurement sites, $\varepsilon_{R}$ is the reduced residual, which is the residual

625 divided by the corresponding standard deviation, $\varepsilon_{R} \sim N(0,1)$.

626 The gradient of $f$ at $x^{0}$ is:

$$
\nabla f_{0}=J\left(x^{0}\right)^{T} W\left(y\left(x^{0}\right)-y^{\text {Meas }}\right)
$$

627 where $\mathrm{W}$ is the weight matrix where the diagonal elements are the reciprocals of the variances of 628 measurement errors; $J\left(x^{0}\right)^{T}=\partial_{x} y\left(x^{0}\right)^{T}$ is the transposed Jacobian matrix of the prediction 629 function at $x=x^{0}$.

630 The Hessian approximation takes the simple form of the symmetrical, positive semi-definite 631 matrix:

$$
H_{0}=J\left(x^{0}\right)^{T} W J\left(x^{0}\right)
$$

632 It is essential for the Jacobian to be full rank of the size of $x$, so that $H_{0}$ is invertible and a definite 633 matrix.

634 An approximation of function $f$ to minimize Eq. (A2) by a quadratic function at $x^{0}$ leads to the 635 approximation of $\mathrm{x}$ :

$$
x=x^{0}-\left(H_{0}\right)^{-1} \nabla f_{0}
$$

636 By replacing Eq. (A2) and Eq. (A3) into Eq. (A5), the approximation of $\mathrm{x}$ can be expressed as:

637 Using Eq. (A1):

$$
x=x^{0}-\left(J\left(x^{0}\right)^{T} W J\left(x^{0}\right)\right)^{-1} J\left(x^{0}\right)^{T} W\left(y\left(x^{0}\right)-y^{\text {Meas }}\right)
$$




$$
x(\varepsilon)=x^{0}+\left(J\left(x^{0}\right)^{T} W J\left(x^{0}\right)\right)^{-1} J\left(x^{0}\right)^{T} W \varepsilon
$$

638 The influence of the measurement errors with regards to the parameter estimates, therefore, can be 639 obtained at the first-order of Eq. (A6):

$$
\Delta x=\left(J\left(x^{0}\right)^{T} W J\left(x^{0}\right)\right)^{-1} J\left(x^{0}\right)^{T} W \varepsilon=\left(W^{\frac{1}{2}} J\left(x^{0}\right)\right)^{\dagger} W^{\frac{1}{2}} \varepsilon=\left(W^{\frac{1}{2}} J\left(x^{0}\right)\right)^{\dagger} \varepsilon_{R}
$$

640 The uncertainty in term of confidence limits can be expressed as:

641

- $\quad$ For $99 \%$ confidence intervals $\left(\left|\varepsilon_{i}\right| \leq 2.58 \sigma_{i}\right)$ :

$$
\begin{gathered}
\|\Delta x\| \leq 2.58\left\|\left(J\left(x^{0}\right)^{T} W J\left(x^{0}\right)\right)^{-1} J\left(x^{0}\right)^{T} W^{\frac{1}{2}}\right\|=2.58\left\|\left(W^{\frac{1}{2}} J\left(x^{0}\right)\right)^{\dagger}\right\| \\
\left|\Delta x_{i}\right| \leq 2.58 \sum_{j=1}^{m}\left|S_{i j}\right|, \text { with } S=\left(W^{\frac{1}{2}} J\right)^{\dagger}
\end{gathered}
$$

- For 95\% confidence intervals $\left(\left|\varepsilon_{i}\right| \leq 1.96 \sigma_{i}\right)$ :

$$
\begin{gathered}
\|\Delta x\| \leq 1.96\left\|\left(W^{\frac{1}{2}} J\left(x^{0}\right)\right)^{\dagger}\right\| \\
\left|\Delta x_{i}\right| \leq 1.96 \sum_{j=1}^{m}\left|S_{i j}\right|, \text { with } S=\left(W^{\frac{1}{2}} J\right)^{\dagger}
\end{gathered}
$$

\section{References}

645 Abe, N \& Peter, B 2010, 'Epanet Calibrator-An integrated computational tool to calibrate hydraulic 646 models', Integrating Water Systems. Boxall \& Maksimovic (eds).

647 Alvisi, S \& Franchini, M 2017, 'Assessment of predictive uncertainty within the framework of 648 water demand forecasting using the Model Conditional Processor (MCP)', Urban Water Journal, vol. 14, no. 1, pp. 1-10.

650 Bargiela, A \& Hainsworth, G 1989, 'Pressure and Flow Uncertainty in Water Systems', Journal of 651 Water Resources Planning and Management, vol. 115, no. 2, pp. 212-229.

652 Beal, C \& Stewart, R 2014, 'Identifying Residential Water End Uses Underpinning Peak Day and 653 Peak Hour Demand', Journal of Water Resources Planning and Management, vol. 140, no. 7, p. 65404014008.

655 Bhave, PR 1988, 'Calibrating water distribution network models', Journal of Environmental 656 Engineering, vol. 114, no. 1, pp. 120-136. 
657 Boulos, PF \& Ormsbee, LE 1991, 'Explicit network calibration for multiple loading conditions', 658 Civil Engineering Systems, vol. 8, no. 3, pp. 153-160.

659 Boulos, PF \& Wood, DJ 1990, 'Explicit calculation of pipe-network parameters', Journal of 660 Hydraulic Engineering, vol. 116, no. 11, pp. 1329-1344.

661 Bush, CA \& Uber, JG 1998, 'Sampling design methods for water distribution model calibration', 662 Journal of Water Resources Planning and Management, vol. 124, no. 6, pp. 334-344.

663 Chin, DA, Mazumdar, A \& Roy, PK 2000, Water-resources engineering, vol. 12, Prentice Hall 664 Englewood Cliffs.

665 Cutore, P, Campisano, A, Kapelan, Z, Modica, C \& Savic, D 2008, 'Probabilistic prediction of 666 urban water consumption using the SCEM-UA algorithm', Urban Water Journal, vol. 5, no. 2, pp. $667 \quad 125-132$.

668 Datta, R \& Sridharan, K 1994, 'Parameter estimation in water-distribution systems by least 669 squares', Journal of Water Resources Planning and Management, vol. 120, no. 4, pp. 405-422.

670 Do, NC, Simpson, AR, Deuerlein, JW \& Piller, O 2016, 'Calibration of Water Demand Multipliers 671 in Water Distribution Systems Using Genetic Algorithms', Journal of Water Resources Planning 672 and Management, p. 04016044.

673 Doucet, A, Godsill, S \& Andrieu, C 2000, 'On sequential Monte Carlo sampling methods for 674 Bayesian filtering', Statistics and computing, vol. 10, no. 3, pp. 197-208.

675 Hol, JD, Schon, TB \& Gustafsson, F 2006, 'On resampling algorithms for particle filters', 676 Nonlinear Statistical Signal Processing Workshop, 2006 IEEE, IEEE, pp. 79-82.

677 Hutton, C, Kapelan, Z, Vamvakeridou-Lyroudia, L \& Savić, D 2013, 'Application of Formal and 678 Informal Bayesian Methods for Water Distribution Hydraulic Model Calibration', Journal of 679 Water Resources Planning and Management, vol. 140, no. 11, p. 04014030.

680 Hutton, CJ \& Kapelan, Z 2015, 'A probabilistic methodology for quantifying, diagnosing and 681 reducing model structural and predictive errors in short term water demand forecasting', 682 Environmental Modelling \& Software, vol. 66, pp. 87-97. 
683 Hutton, CJ, Kapelan, Z, Vamvakeridou-Lyroudia, L \& Savic, DA 2012a, 'Real-time demand estimation in water distrubtion systems under uncertainty ', paper presented at WDSA 2012: 14th Water Distribution Systems Analysis Conference.

686 Hutton, CJ, Kapelan, Z, Vamvakeridou-Lyroudia, L \& Savić, DA 2012b, 'Dealing with uncertainty 687 in water distribution system models: A framework for real-time modeling and data assimilation', Journal of Water Resources Planning and Management, vol. 140, no. 2, pp. 169-183.

Kang, D \& Lansey, K 2009, 'Real-time demand estimation and confidence limit analysis for water

691 Kapelan, ZS, Savic, DA \& Walters, GA 2007, 'Calibration of water distribution hydraulic models

692 using a Bayesian-type procedure', Journal of Hydraulic Engineering, vol. 133, no. 8, pp. 927-936.

693 Kitagawa, G 1996, 'Monte Carlo filter and smoother for non-Gaussian nonlinear state space 694 models', Journal of computational and graphical statistics, vol. 5, no. 1, pp. 1-25.

695 Lansey, K, El-Shorbagy, W, Ahmed, I, Araujo, J \& Haan, C 2001, 'Calibration assessment and 696 data collection for water distribution networks', Journal of Hydraulic Engineering, vol. 127, no. 697 4, pp. 270-279.

698 Lansey, KE \& Basnet, C 1991, 'Parameter estimation for water distribution networks', Journal of 699 Water Resources Planning and Management, vol. 117, no. 1, pp. 126-144.

700 Moradkhani, H, Hsu, KL, Gupta, H \& Sorooshian, S 2005, 'Uncertainty assessment of hydrologic 701 model states and parameters: Sequential data assimilation using the particle filter', Water 702 Resources Research, vol. 41, no. 5.

703 Nagar, AK \& Powell, RS 2002, 'LFT/SDP approach to the uncertainty analysis for state estimation 704 of water distribution systems', Control Theory and Applications, IEE Proceedings-, IET, vol. 149, 705 pp. $137-142$.

706 Nicklow, J, Reed, P, Savic, D, Dessalegne, T, Harrell, L, Chan-Hilton, A, Karamouz, M, Minsker, 707 B, Ostfeld, A, Singh, A \& Zechman, E 2010, 'State of the Art for Genetic Algorithms and Beyond 708 in Water Resources Planning and Management', Journal of Water Resources Planning and 709 Management, vol. 136, no. 4, pp. 412-432. 
710 Ormsbee, LE 1989, 'Implicit network calibration', Journal of Water Resources Planning and 711 Management, vol. 115, no. 2, pp. 243-257.

712 Ormsbee, LE \& Wood, DJ 1986, 'Explicit pipe network calibration', Journal of Water Resources 713 Planning and Management, vol. 112, no. 2, pp. 166-182.

714 Ostfeld, A, Salomons, E, Ormsbee, L, Uber, JG, Bros, CM, Kalungi, P, Burd, R, Zazula-Coetzee, 715 B, Belrain, T \& Kang, D 2011, 'Battle of the water calibration networks', Journal of Water 716 Resources Planning and Management, vol. 138, no. 5, pp. 523-532.

717 Perelman, L, Maslia, ML, Ostfeld, A \& Sautner, JB 2008, 'Using aggregation/skeletonization 718 network models for water quality simulations in epidemiologic studies', Journal (American Water 719 Works Association), vol. 100, no. 6, pp. 122-133.

720 Piller, O 1995, 'Modeling the behavior of a network-Hydraulic analysis and sampling procedures 721 for parameter estimation', Applied Mathematics thesis from the University of Bordeaux (PRES), 722 vol. $\mathrm{PhD}$ Thesis.

723 Preis, A, Whittle, A \& Ostfeld, A 2009, 'Online hydraulic state prediction for water distribution 724 systems', World Environmental and Water Resources Congress 2009: Great Rivers, May 17, 2009 725 - May 21, 2009, American Society of Civil Engineers, Kansas City, MO, United states, vol. 342, 726 pp. 323-345.

727 Preis, A, Whittle, AJ, Ostfeld, A \& Perelman, L 2010, 'On-line hydraulic state estimation in urban 728 water networks using reduced models', 10th International Conference on Computing and Control 729 for the Water Industry: Integrating Water Systems, CCWI 2009, September 1, 2009 - September 730 3, 2009, CRC Press, Sheffield, United kingdom, pp. 319-324.

731 Preis, A, Whittle, AJ, Ostfeld, A \& Perelman, L 2011, 'Efficient Hydraulic State Estimation 732 Technique Using Reduced Models of Urban Water Networks', Journal of Water Resources 733 Planning and Management, vol. 137, no. 4, pp. 343-351.

734 Rahal, CM, Sterling, MJH \& Coulbeck, B 1980, 'Parameter tuning for simulation models of water 735 distribution networks', Proceedings of the Institution of Civil Engineers, vol. 69, no. 3, pp. 751736762

737 Ristic, B, Arulampalm, S \& Gordon, N 2004, Beyond the Kalman filter : particle filters for 738 tracking applications, Artech House Boston, Ma., London. 
Rossman, LA 2000, 'EPANET 2: users manual', US Environmental Protection Agency (EPA), USA.

Salmond, D \& Gordon, N 2005, 'An introduction to particle filters', State space and unopbserved component models theory and applications, pp. 1-19.

Sanz, G \& Pérez, R 2014, 'Comparison of demand pattern calibration in water distribution networks with geographic and non-geographic parameterization', 11th International Conference on Hydroinformatics, pp. 1-8.

Sanz, G \& Pérez, R 2015, 'Sensitivity analysis for sampling design and demand calibration in water distribution networks using the singular value decomposition', Journal of Water Resources Planning and Management, vol. 141, no. 10, p. 04015020.

Savic, DA, Kapelan, ZS \& Jonkergouw, PM 2009, 'Quo vadis water distribution model calibration?', Urban Water Journal, vol. 6, no. 1, pp. 3-22.

Shang, F, Uber, JG, Van Bloemen Waanders, BG, Boccelli, D \& Janke, R 2006, 'Real time water demand estimation in water distribution system', 8th Annual Water Distribution Systems Analysis Symposium 2006, August 27, 2006 - August 30, 2006, American Society of Civil Engineers, Cincinnati, OH, United states, 10.1061/40941(247)95, p. 95.

Thyer, MA, Micevski, T, Kuczera, GA \& Coombes, P 2011, 'A behavioural approach to stochastic end use modelling', paper presented at Ozwater 11, Australia's National Water Conference and Exhibition.

USEPA 2005, Water Distribution System Analysis: Field Studies, Modeling and Management. A Reference Guide for Utilities, O USEPA Cincinnati, USA, USEPA Cincinnati, Ohio, USA.

van Leeuwen, PJ 2009, 'Particle filtering in geophysical systems', Monthly Weather Review, vol. 137, no. 12 , pp. 4089-4114.

van Leeuwen, PJ 2010, 'Nonlinear data assimilation in geosciences: an extremely efficient particle filter', Quarterly Journal of the Royal Meteorological Society, vol. 136, no. 653, pp. 1991-1999. van Zyl, JE, Piller, O \& le Gat, Y 2008, 'Sizing municipal storage tanks based on reliability criteria', Journal of Water Resources Planning and Management, vol. 134, no. 6, pp. 548-555. 
766 Waller, JA 2013, 'Using observations at different spatial scales in data assimilation for 767 environmental prediction', PhD Thesis, Department of Mathematics and Statistics, University of 768 Reading. Online at: www. reading. ac. uk/maths-and-stats/research/theses/maths-phdtheses. aspx.

769 Walski, TM 1983, 'Technique for calibrating network models', Journal of Water Resources 770 Planning and Management, vol. 109, no. 4, pp. 360-372.

771 Weerts, AH \& El Serafy, GY 2006, 'Particle filtering and ensemble Kalman filtering for state 772 updating with hydrological conceptual rainfall-runoff models', Water Resources Research, vol. 42, 773 no. 9.

$774 \mathrm{Xu}, \mathrm{C} \&$ Goulter, IC 1998, 'Probabilistic model for water distribution reliability', Journal of Water 775 Resources Planning and Management, vol. 124, no. 4, pp. 218-228. 
776

777

Table 1: Coefficient of determination $\left(R^{2}\right)$ and root mean squared error (RMSE) of demand estimates corresponding to different

\begin{tabular}{ccccc}
\hline \multirow{2}{*}{ No } & $\begin{array}{c}\text { Auto-regression coefficient } \\
(\phi)\end{array}$ & $\begin{array}{c}\text { Variance of demand residual } \\
\left(\sigma_{h}^{2}\right)\end{array}$ & $\mathrm{R}^{2}$ & RMSE \\
\hline 1 & & 0.04 & 0.465 & 0.198 \\
2 & 0.3 & 0.25 & 0.986 & 0.030 \\
3 & & 0.64 & 0.983 & 0.033 \\
4 & & 0.04 & 0.528 & 0.189 \\
5 & 0.5 & 0.25 & 0.986 & 0.030 \\
6 & & 0.64 & 0.987 & 0.029 \\
7 & & 0.04 & 0.982 & 0.033 \\
8 & 0.7 & 0.25 & $\mathbf{0 . 9 8 8}$ & $\mathbf{0 . 0 2 8}$ \\
9 & & 0.64 & 0.986 & 0.031 \\
10 & & 0.04 & 0.987 & 0.029 \\
11 & 0.9 & 0.25 & 0.986 & 0.031 \\
12 & & 0.64 & 0.985 & 0.031 \\
\hline
\end{tabular}

778

*Bold - Best estimated result

779

780

781

\begin{tabular}{c|cc|cc|cc}
\hline Model type & \multicolumn{2}{|c|}{ DMFLive-I } & \multicolumn{2}{c}{ DMFLive-II } & \multicolumn{2}{c}{ DMFLive-III $\left(N_{G e n}=50\right)$} \\
No. Particles & $\mathrm{N}_{\mathrm{P}}=1000$ & $\mathrm{~N}_{\mathrm{P}}=5000$ & $\mathrm{~N}_{\mathrm{P}}=1000$ & $\mathrm{~N}_{\mathrm{P}}=5000$ & $\mathrm{~N}_{\mathrm{P}}^{\mathrm{GA}}=20$ & $\mathrm{~N}_{\mathrm{P}}^{\mathrm{GA}}=100$ \\
No. Eval. & $1.43^{*} 10^{5}$ & $7.15^{*} 10^{5}$ & $1.43^{*} 10^{5}$ & $7.15^{*} 10^{5}$ & $1.08 * 10^{5}$ & $5.43^{*} 10^{5}$ \\
\hline RMSE $_{\text {DMF1 }}$ & 0.386 & 0.405 & 0.050 & 0.027 & 0.107 & 0.030 \\
RMSE $_{\text {DMF2 }}$ & 0.365 & 0.422 & 0.026 & 0.021 & 0.067 & 0.025 \\
RMSE $_{\text {DMF3 }}$ & 0.416 & 0.237 & 0.029 & 0.027 & 0.068 & 0.023 \\
RMSE $_{\text {DMF4 }}$ & 0.385 & 0.229 & 0.043 & 0.038 & 0.086 & 0.032 \\
RMSE $_{\text {DMF5 }}$ & 0.366 & 0.246 & 0.074 & 0.049 & 0.190 & 0.050 \\
\hline
\end{tabular}

Table 2: Actual and estimated total volume of water usage during calculated period

\begin{tabular}{lccc}
\hline \multicolumn{1}{c}{ Cases } & $\begin{array}{c}\text { Total } \\
\left(\mathrm{m}^{3} / \text { day }\right)\end{array}$ & $\begin{array}{c}\text { Difference } \\
\left(\mathrm{m}^{3} / \text { day }\right)\end{array}$ & $\begin{array}{c}\text { \% Difference } \\
(\%)\end{array}$ \\
\hline Actual daily water usage & 5989.25 & & \\
Estimated water usage with DMFLive & 5942.43 & 46.81 & 0.78 \\
Estimated water usage with DMF-WTLive & 6007.31 & 18.07 & 0.30 \\
\hline
\end{tabular}

18.07

0.78

(1) 